\title{
BMJ Open Metro and elderly health in Hong Kong: protocol for a natural experiment study in a high-density city
}

\author{
Guibo Sun (1D , ${ }^{1}$ Yao Du, ${ }^{1}$ Michael Y Ni, ${ }^{1,2,3}$ Jianting Zhao, ${ }^{1}$ Chris Webster ${ }^{1}$
}

To cite: Sun G, Du Y, Ni MY, et al. Metro and elderly health in Hong Kong: protocol for a natural experiment study in a high-density city. BMJ Open 2021;11:e043983. doi:10.1136/ bmjopen-2020-043983

- Prepublication history for this paper is available online. To view these files, please visit the journal online (http://dx.doi org/10.1136/bmjopen-2020043983).

Received 22 August 2020 Revised 03 February 2021 Accepted 26 February 2021

\section{Check for updates}

C Author(s) (or their employer(s)) 2021. Re-use permitted under CC BY-NC. No commercial re-use. See rights and permissions. Published by BMJ.

${ }^{1}$ Healthy High Density Cities Lab, Faculty of Architecture, The University of Hong Kong, Hong Kong, China

${ }^{2}$ School of Public Health, Li Ka Shing Faculty of Medicine, The University of Hong Kong, Hong Kong, China

${ }^{3}$ The State Key Laboratory of Brain and Cognitive Sciences, The University of Hong Kong, Hong Kong, China

Correspondence to

Dr Guibo Sun; gbsun@hku.hk

\begin{abstract}
Introduction Public transport accessible to older people may offer a transformative solution to achieving healthy ageing. However, the evidence to support such transport infrastructure modifications is unclear. Previous studies on public transport use and elderly health were mostly observational studies using cross-sectional data. Few studies have examined the before-and-after effects of a new metro, for example, to see if it leads to improved elderly health.
\end{abstract}

Methods and analysis We use a new metro line in Hong Kong as a natural experiment to examine the impact of the metro-led public transport intervention on elderly health. In Hong Kong, more than $90 \%$ of daily travels are made by public transport. The public transport modifications consist of the new metro line with eight stations and changes in the walking environment and bus services around the stations. We will look at the before-and-after differences in public transport use and health outcomes between elderly participants living in treatment neighbourhoods ( $400 \mathrm{~m}$ walking buffered areas of the new metro stations) and in control groups (living in comparable areas but unaffected by the new metro). Questionnaire-based baseline data were collected in 2019 before the COVID-19 pandemic, while some qualitative interviews are ongoing. Amid the pandemic, we conducted a quick telephone-based survey of COVID-19's potential impact on public transport use behaviours of our elderly cohort in September 2020. Note there is no lockdown in Hong Kong until the writing of the paper (January 2021). After the new metro opens, we will conduct a follow-up survey, tentatively in late 2022. We aim to investigate if the new metro and the associated changes in the built environment have any effects on public transport use behaviours, physical activity and wider health outcomes among the elderly (eg, social inclusion, quality of life, subjective well-being)

Ethics and dissemination The Human Research Ethics Committee of the University of Hong Kong reviewed and approved the study procedures and materials (reference number: EA1710040). Results will be communicated through scientific papers and research reports.

\section{INTRODUCTION}

Public transport use may influence physical activity, social inclusion and well-being of the elderly. ${ }^{1}$ The rapid growth in the ageing population poses a great challenge to offering travel options that consider the

\section{Strengths and limitations of this study}

- This study will investigate how the new metro and the associated changes in the built environments can be used as a source of public health interventions for the elderly.

- We investigate a more specific set of research questions linking the new metro and the associated changes in built environment with public transport use, physical activity and wider health impact among the elderly cohort in a high-density city.

- Our natural experiment research design with multiple treatment-control groups will provide robust estimates of the health impact of public transport.

- Although the natural experiment research design is insightful, this type of large-scale study may encounter financial and time constraints due to risk of postponement and other uncertainties (eg, COVID-19) during the infrastructure modifications; hence, research design needs to be adaptive.

usage patterns of the elderly. As people get older, without specific interventions aimed at the elderly, there is a gap between demand and supply for public transport. For example, in Hong Kong, an ageing society, public transport focuses on expanding its metro system to sustain the high-density city. However, a travel characteristics survey revealed that elders are reluctant to use the metro. ${ }^{2}$ This demandsupply gap in providing public transport for the elderly is becoming a problem in achieving healthy ageing. ${ }^{34}$

However, we do not have much scientific evidence to support transport infrastructure modifications that improve health outcomes of older people. The associations between public transport use and elderly health effects are mostly based on observational studies with cross-sectional data. Few studies have examined the before-and-after effects of a new metro, for example, to see if it leads to meaningful health improvements for the older people. ${ }^{56}$ We cannot manipulate transport modifications experimentally to test 
what works and how it works; the public transport system changes are usually out of researchers' control. ${ }^{7}$

A natural experiment can use public transport changes that 'naturally occurred', such as a new bus route, to examine health effects among older people. ${ }^{8}$ Studies are emerging in using built environment changes as public health interventions. ${ }^{9}{ }^{10}$ A few review papers include a broad range of built environment changes such as park improvements, new walking and cycling greenways, busways and light rail transit. ${ }^{11-16}$ They found that new public bus and light rail transit may encourage uptake of active travel, and participants who adopted the new transport have better health performance (eg, physical activity and well-being). ${ }^{17-20}$ For example, Ogilvie and colleagues ${ }^{17-20}$ examined the travel behaviours and their health impact among working commuters using a natural experiment of a new bus line, Cambridgeshire Guided Busway. Their studies provided insights that a new busway might be associated with an increase in the use of active travel modes on the commuting journey and better mental health among users. However, the relationship was rather complicated. ${ }^{20-23}$ Designated treatmentcontrol groups were both exposed to the new busway, and participants' retention ratio was relatively low in the follow-up survey partially because the busway opening was delayed for more than 2 years. ${ }^{20}{ }^{21}$ A longitudinal study in Seattle, with only treatment groups included, found that light rail transit leads to increased walking activities in station areas. ${ }^{22}$ Panel data were also used to investigate the impact on walking and physical activity before and after a new light rail transit line in California. A differential effect of public transit on active travel was revealed in a quasi-experimental design; for example, sedentary groups were affected more than the others. ${ }^{23}$ Both the light rail transit studies had relatively small sample sizes (eg, 73 participants in a follow-up survey), limiting the capacity to infer the complicated effects of the interventions. The existing natural experiment studies on public transport use and health effects focused on adult groups, public bus or light rail transit as the transport modifications, or health outcomes relating to work commuting behaviours. More importantly, most natural experiment studies were in low-density cities. ${ }^{7}$

We present a natural experiment study of metro and elderly health in Hong Kong to investigate the plausible effects of the new metro and the associated changes in the built environment on public transport use, physical activity and wider health outcomes among older people. Our aim is not to evaluate the new metro against a comprehensive set of health outcomes, but rather to use the opportunity presented by this natural experiment to investigate a more specific set of research questions linking associated changes in built environment with changes in public transport use, physical activity and wider health outcomes among older people in high-density cities. The study will address a few issues in previous research ${ }^{724-28}$ : (1) There is a lack of longitudinal, treatment-control research design that investigates causal inference between a new metro, built environment changes and elderly health. (2) There is a lack of accurate measures of built environment and its changes related to public transport use in highdensity cities. (3) There is a lack of research that takes a holistic view of qualitative and quantitative data to investigate metro-led transport infrastructure modifications and their health effects among older people.

\section{Research objectives}

Main research objective

- We aim to investigate if the new metro and the associated changes in the built environment have any effects on public transport use and wider health outcomes among a cohort of elderly in Hong Kong.

\section{Secondary research questions/objectives}

- Has the new metro changed the public transport mode share of the elderly?

- What are the built environment characteristics related to the uptake of (or decrease in) active travel and related physical activity of the elderly?

- What is the impact of changes in public transport use on wider health outcomes (eg, social inclusion, quality of life, mental health and subjective well-being)?

\section{METHODS AND ANALYSIS}

\section{Overall research design}

\section{Quantitative cohort}

- A cohort of older people (age $\geq 65$ ) to study the changes in public transport use behaviours, physical activity and wider health outcomes, using repeated interviewer-administrated questionnaires and objective measurements (eg, accelerometer, map, walking diary).

From the quantitative cohort, we invite a proportion of participants to form a qualitative cohort:

- A semistructured interview on the attitudes and concerns towards the new metro, the associated changes in the built environment and bus service adjustment, and how the interventions would influence their public transport use and wider health behaviours.

- A go-along interview of the walking environment of routes to bus stops and metro stations before and after the new metro.

\section{Setting: Hong Kong}

Hong Kong is one of the most densely populated places in the world. Over seven million people live within $1068 \mathrm{~km}^{2}$, but more than $75 \%$ of the land is non-built-up area. Additionally, the population is rapidly ageing. In 2018, older people aged 65 or above counted towards $17.9 \%$ of the population and are expected to reach $31.9 \%$ by $2038 .{ }^{29}$

The city focuses on using a metro system to sustain the high-density built environment. Around half of the population live within $500 \mathrm{~m}$ from a metro station. The metro carries an average of 4.69 million passengers per day. ${ }^{30}{ }^{31}$ The system is also expanding: three line extensions entered into operation between 2015 and 2016, and 
the metro expanded from $212 \mathrm{~km}$ with 84 stations in 2007 to over $270 \mathrm{~km}$ with 98 stations in 2019 . The government plans to double its size. With the continued expansion of the metro system, bus services are being cancelled, reduced or truncated because transport planning in Hong Kong uses buses as the metro's rider feeders. ${ }^{32}$

Hong Kong is a three-dimensional (3D) city with highrises connected by elevated walkways and undergrounds. ${ }^{33}$ Walking and public transport accessibilities are shaped by a 3D pedestrian network, ${ }^{31} 3435$ which connects over 2000 footbridges, 400 tunnels and $60 \mathrm{~km}$ walking underground at all metro stations. ${ }^{31}$ It means that even if living at a metro station entrance above ground, older people may still need to walk more than 10 min underground to access the train.

\section{Intervention}

The intervention includes (1) a new metro line with eight stations, of which half are newly built and the rest as modifications of the existing ones by adding new platforms, exits and transfer connections; (2) associated changes in the built environment, such as 3D pedestrian networks, open spaces and amenities around the stations; and (3) changes in nearby bus services.

The new metro line was scheduled to open in May 2019, but concrete structures of the newly built platforms at Hung Hom station failed the safety inspection. The line was thus postponed. The government decided that the new line will open in two phases (figure 1 ). The first phase was in February 2020, when four stations were opened, with the second phase being postponed to late 2021 after the reinforcement work at Hung Hom is completed.

\section{Comparisons: the treatment-control groups}

Figure 2 shows the treatment-control groups for investigating the effects of the interventions. Paired treatment-control groups are based on comparability in neighbourhood types, regional accessibility, socioeconomic status and demographics. Three treatment groups were picked from the new line (groups 1-T, 2-T and 3-T). Participants are older people living within a neighbourhood around the treatment station exits, delineated by a $400 \mathrm{~m}$ buffer zone from the exits, based on the pedestrian network. This selection method ensures that they will experience the changes brought by a new station. We selected three control groups (groups 1-C, 2-C and 3-C). Participants in the control groups live in comparable station catchments, but the metro stations have been in operation for more than 5 years. We aim to conduct a difference-in-difference analysis between the treatment and control groups by validating that confounders varying across the groups are time-invariant, and timevarying confounders are group-invariant. ${ }^{36}$

We will also conduct a longitudinal study of another two lines that entered into operation recently. Sites were selected from sections between Sheung Wan and Kennedy Town stations of the Island Line (opened in March 2015, group 1-E) and from Admiralty to South Horizons stations

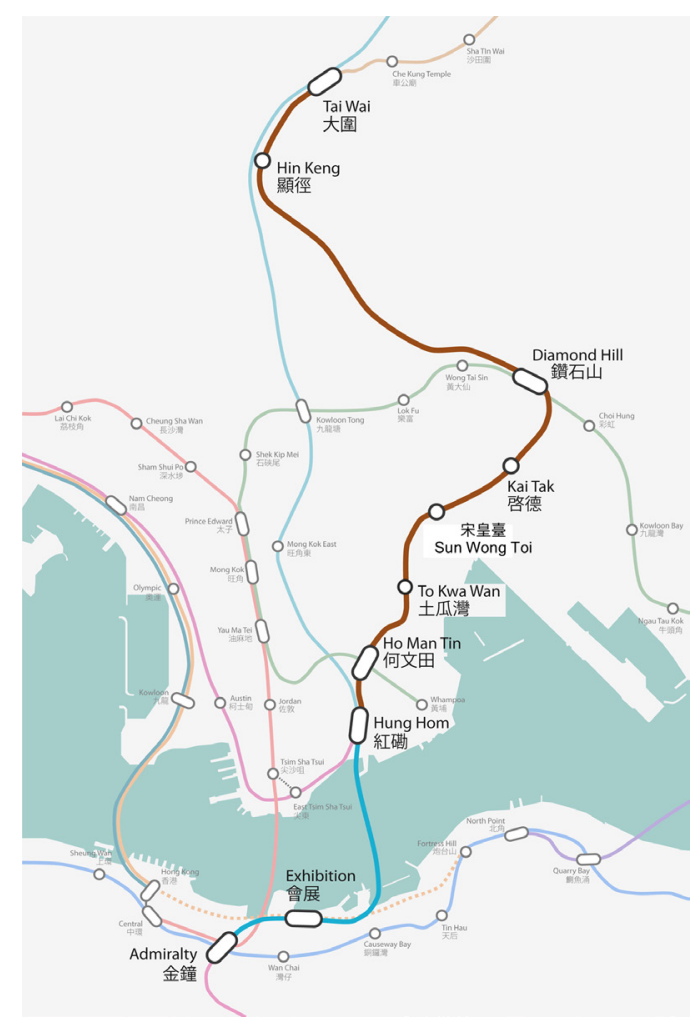

Figure 1 The new metro line from Tai Wai to Hung Hom stations. The line will open in two phases: the first phase was in February 2020 with four stations opened (Tai Wai, Hin Keng, Diamond Hill and Kai Tak); the second phase will be in 2021 (including Sung Wong Toi, To Kwa Wan, Ho Man Tin and Hung Hom stations). The map was adapted under free art licence from Wikimedia. Retrieved from https://commons. wikimedia.org/wiki/File:SCL_ga_map.png.

of the South Island Line (opened in December 2016, group 2-E). Their comparable groups are 4-C and 1-C, respectively. Besides the survey instruments we describe in the following sections, we will also ask participants to recall their travel behaviours before metro extensions in 1-E and 2-E.

Questionnaire-based baseline data were collected in 2019 before the COVID-19 pandemic, while some qualitative interviews are ongoing. Amid the pandemic, we conducted a quick telephone-based survey of COVID19 's potential impact on public transport use behaviours among our elderly cohort in September 2020. Note there is no lockdown in Hong Kong until the writing of the paper (January 2021). After the new metro opens, we will conduct a follow-up survey, tentatively in late 2022.

\section{Participants}

Inclusion criteria

Participants are (1) 65 years of age or older, (2) Cantonese-speaking, (3) residents in the neighbourhoods for at least 3 years (to control potential selfselection bias $^{37}$ ) and (4) able to walk independently for at least $15 \mathrm{~min}$. 


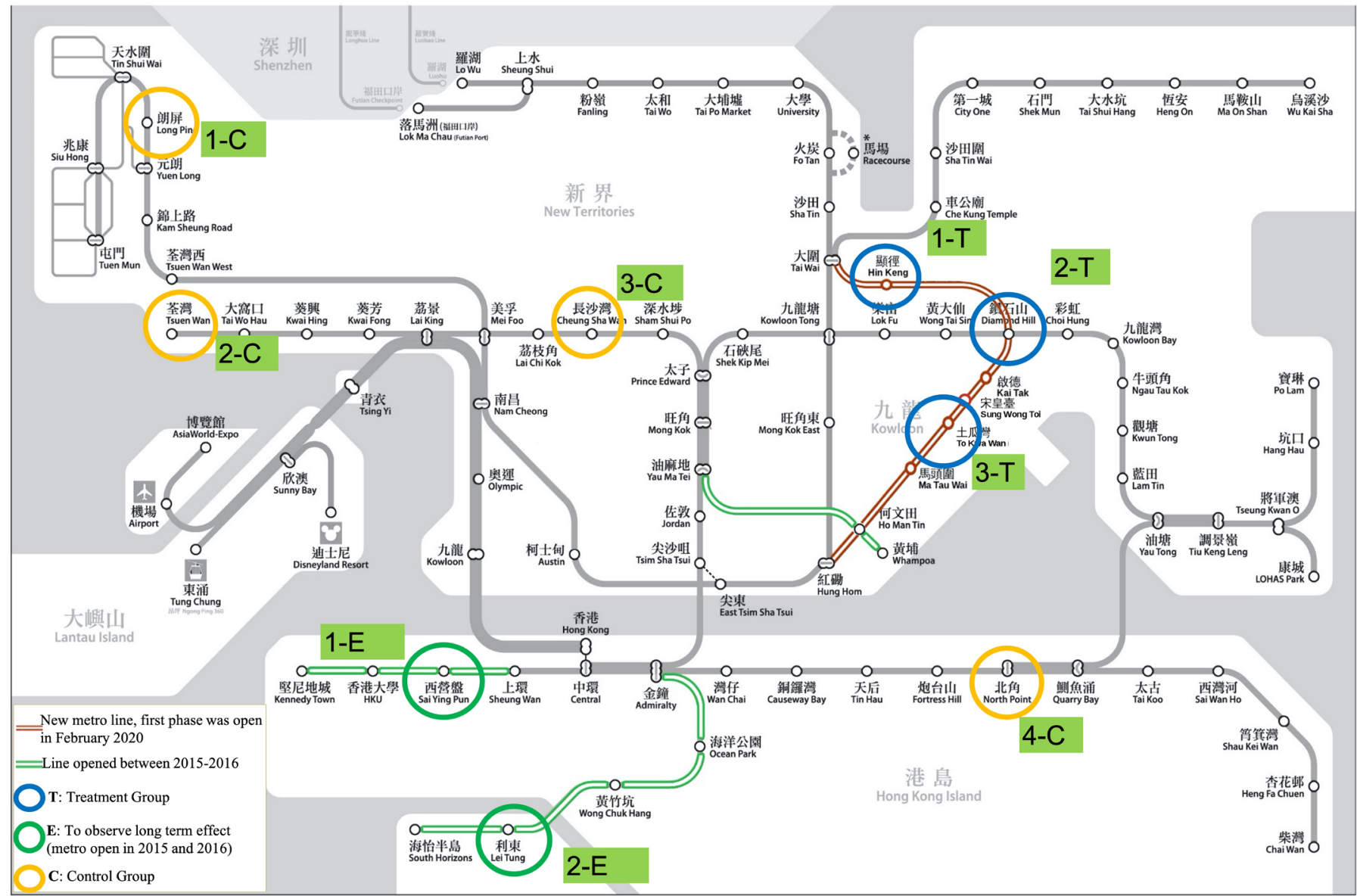

Figure 2 Treatment-control groups represent pairing treatment-control groups for investigating the health impact of public transport among the elderly. Three treatment groups are selected from the new line (groups 1-T, 2-T and 3-T), compared with control groups (groups 1-C, 2-C and 3-C) from lines in operation for more than 5 years. Groups 1-E and 2-E are selected from two lines that entered into operation in March 2015 and December 2016, and their comparable groups are 4-C and 1-C. The background metro map was adapted under Creative Commons Attribution ShareAlike 3.0 from Wikimedia. Retrieved from https://upload.wikimedia.org/wikipedia/commons/f/f1/Shatin_to_Central_Link_proposal_final.svg.

\section{Recruitment}

Participants were recruited from the neighbourhood elderly centres (NEC) located within the study areas indicated in figure 2. In Hong Kong, 170 NECs cover the territory to provide government community services for the elderly at the neighbourhood level. Elderly centre-based recruitment can guarantee retention rate for follow-up surveys. ${ }^{25}$ The principal investigator (PI) visited the NECs to brief on the research design and got the centre's agreement to participate in the research. We used quota sampling, with considerations of age distribution, gender balance and home locations, to systematically recruit participants from the centre's database. An NEC could have more than 800 members. Invitations will be sent both by phone call and during the centre's monthly gathering events. The NEC helped verify if the candidates meet our criteria, and the PI and the research assistants conducted further scrutiny before data collection.

Each questionnaire survey or interview will take around 1 hour. A modest incentive of HK $\$ 50$ was provided to participants after completing the baseline questionnaire survey. The elders participating in the go-along interview or accelerometer component will receive an extra HK $\$ 50$. To boost the retention rate for the follow-up survey, another HK $\$ 100$ will be provided on completion of the follow-up survey.

\section{Sample size estimation}

To ensure the explanatory power of the panel data models, we aim to achieve a cohort of 720 elderly participants for the main questionnaire survey. It can provide $80 \%$ power to detect a significant difference between the treatment and control groups, and 0.05 significance levels of statistical modelling with multiple $(>12)$ built environment independent variables. The retention rate is expected to be $70 \%-80 \%$. We thus anticipate a need to obtain a baseline survey of 900 participants, with each of the nine study sites recruiting 100 participants. We aim to get the following age distributions: $65-74$ years: $75 \%$; 75-84 years: $20 \%$; $85+$ years: $5 \%$.

All participants will join the main questionnaire survey, with a proportion of them being invited to the qualitative interview. The interview involves semistructured interviews ( $\mathrm{n}=135$ participants) to assess mental health 
and well-being effects and go-along interviews $(n=60)$ to conduct walking environment situational analysis. We will consider an even distribution of participants' gender, age range, home location, travel mode preference and health conditions.

The follow-up study will be conducted in similar forms as the baseline, and participants will be contacted for the follow-up survey assisted by the NECs.

\section{Patient and public involvement \\ No patients were involved.}

\section{Core questionnaire survey}

Travel behaviours, social norms and attitudes, and covariates

For general travel behaviours, we will:

- Collect the most frequently used travel mode, frequent destinations and self-efficacy of using each mode (including walking, bus, minibus, metro, tram, light rail and car).

For travel mode share and route environment to stop/ station of each travel mode, we will:

- Collect the usage frequency of each travel mode, mostly used stops/stations, travel destinations, walking time to the stops/stations, sidewalk condition, crowdedness and other barriers (in Likert scale).

- Ask open questions on the pros and cons of using each travel mode.

For perceived neighbourhood environment and 3D walking environment, we will:

- Ask questions adapted from the Neighborhood Environment Walkability Scale for Chinese Seniors ${ }^{38}$ on the walking condition, route choice, sidewalk width, lighting, shelter, traffic, speed, safety and road crossing (in Likert scale), and other concerns (open question).

- Measure social inclusiveness and discriminations when using metro.

- Collect data on perceived difficulties in using the footbridge, metro underground path and pedestrian facilities for elderly (eg, resting areas, escalators, moving walkaway and lift); two questions are about bus stop environment on bench and shelter provisions, and other concerns (open question).

\section{Covariates}

Covariates include self-rated economic condition, health status (chronic diseases and types) and their perceived impact on mobility and sociodemographics.

\section{Wider health outcomes}

For physical activity and walking diary, we will:

- Use the Chinese version of the International Physical Activity Questionnaire-Short (past 7 days) for the elderly to measure weekly minutes of physical activity and daily minutes of sitting.

- Provide accelerometers (ActiGraph GT3X+) for those elders who wish to wear it for 7 days (estimating a quarter of the sample) to measure the intensity of physical activity and sedentary behaviours.
- Maintain a 7-day walking diary to record the time, duration, origin and destination of each trip, and activities when participants are wearing the accelerometers.

For health-related quality of life and depressive symptoms, we will:

- Use the Short-Form Health Survey-12 scale to assess general physical and mental health.

- Use Patient Health Questionnaire-9 to assess depressive symptoms.

For social network and loneliness, we will:

- Use the Lubben Social Network Scale-6 to measure social networking.

- Use the University of California, Los Angeles, Loneliness Scale -Version 3 (UCLA LS3) to measure loneliness.

For subjective well-being, we will:

- Measure three aspects of subjective well-being, ${ }^{39}$ namely evaluative well-being (life satisfaction), hedonic well-being (feelings of happiness, sadness, anger, stress and pain) and eudaimonic well-being (a sense of purpose and meaning in life).

Evaluative well-being will be measured using the 'Cantril Ladder'. ${ }^{40}$

Hedonic well-being will be measured using the Scale of Positive and Negative Experience, assessing subjective feelings of well-being and ill-being. This 12-item questionnaire includes 6 items to assess positive feelings and 6 items to determine negative feelings. For both positive and negative items, three of the items are generic (eg, positive, negative) and three subscales are more specific (eg, joyful, sad). ${ }^{41}$

Eudaimonic well-being will be measured using the 18-item scale of the 6 dimensions of well-being, namely self-acceptance, purpose in life, personal growth, positive relations with others, environmental mastery and autonomy. ${ }^{42}$

\section{Built environment changes: measures and 3D pedestrian network data}

We will use geographical information system to measure changes in neighbourhood and route environments. We will design indicators related to the neighbourhood's density, diversity, design, destination accessibility and distance to transit using the complete built environment data collected from the Buildings Department, Lands Department and Transport Department in Hong Kong. ${ }^{43}$ In addition, we will incorporate geocoded amenities data to enrich the built environment indicators. We have extracted such data from points of interest (POI) data sources and will retrieve time-series POIs (eg, 329644 amenities in 2017) from a location-based navigation company. The government amenities data will validate the third-party data.

Route environment will be modelled on the 3D pedestrian network. ${ }^{31}$ Our Hong Kong 3D pedestrian network data (segments=269242) include 23 types of pedestrian paths, encompassing 5 height levels: surface level (eg, sidewalk), underground paths (eg, metro stations), 
footbridge, underground linkage level that connects surface and underground paths, and above-ground linkage level that connects surface and footbridges. The linkage infrastructure for all levels includes stairs, ramps, escalators and elevators. The 3D pedestrian network data enable us to gather various route options available to pedestrians when accessing public transport in the highdensity city of Hong Kong.

\section{Qualitative data}

We will use qualitative data to understand and address multiple facets of the metro-led public transport interventions. Previous studies suggested that relying only on quantitative data would be insufficient for elucidating the understanding of how the interventions and associated built environment changes matter for elderly health. ${ }^{20} 44$ We will use semistructured interviews to investigate the potential effects of metro interventions on elderly health. In the baseline interview, we will explore participants' experience using different travel modes, mode choice rationales and effects on their mental health and wellbeing. In the follow-up interview, we will examine how and why participants' travel behaviours, mental health and well-being have been changed by the metro-led public transport intervention and evaluate the potential impact pathways of the changes. In addition, micro-scale characteristics of the route environment when approaching public transport are context-specific. ${ }^{45}$ Therefore, we will apply a go-along interview, ${ }^{46}$ a mobile method to understand interactions between individual and built environment interventions. ${ }^{47} 48$ We will collect concerns, walking barriers and areas of improvement from older people's views by walking along with participants on predesigned walking paths towards metro stations and bus stops. We aim to obtain detailed and context-specific information concerning the elderly to produce insights into how and why the interventions matter. ${ }^{49-51}$

\section{Analysis}

We will use various analysis methods to improve our understanding of the changing patterns of public transport use behaviours in our cohort before and after the metro intervention, investigating the impact of the new metro and associated changes in built environment on wider health outcomes.

\section{Qualitative analysis}

We will apply content analysis to organise and elicit qualitative data. ${ }^{52}$ Cantonese will be the language in the interview. Audio records will be translated into English scripts. We will use built-in coding and analysis tools in NVivo (V.12) to extract word frequency and topic intersection. Recurring ideas in the scripts can be obtained as counts in the word frequency analysis. We will read the transcript carefully to investigate recurring topics and ideas. We will design a framework to analyse the interview scripts. We will also extract participants' quotes and present them according to the analysis framework. ${ }^{51}$ The qualitative results will work with quantitative ones to investigate the new metro's health effects among older people.

\section{Quantitative analysis}

We will use difference-in-difference analysis to study the public transport use behaviours and wider health outcomes between the treatment and control groups before and after the intervention periods. We will integrate data to strengthen causal inferences of the natural experiment, such as using multiple pre-post measures to control for singular changes and multiple treatmentcontrol groups that differ according to some variables.

We will also use multivariable regression models to estimate the associations between dependent and independent variables. We will build up these models by progressively adjusting them for various individual, geographical and covariates that potentially confound the relationships. We will stratify some models by prespecified effect modifiers, for example, by gender and age in the analysis of the correlates of physical activity and wider health outcomes, and by baseline public transport use behaviour in the longitudinal data analysis. Several relationships will be tested. For example:

- Changes in route environment: changes in public transport use. We will examine the effects of the 3D pedestrian network (eg, elements, lengths), neighbourhood walkability and perceived difficulties of walking in the $3 \mathrm{D}$ city on public transport use behaviours.

- Changes in public transport mode: changes in physical activity. We will examine how the different mode choice and use would impact physical activity, controlling objective and perceived route environment and distance to station/stops.

- Changes in physical activity: changes in quality of life and subjective well-being. We will investigate how physical activity related to public transport use impacts physical and mental health and subjective well-being, controlling public transport mode choice and use.

- Changes in social inclusion: changes in mental health and depressive symptom. We will examine how the social inclusion enabled by public transport impacts mental health and depressive symptoms by controlling public transport use and social environment.

\section{ETHICS AND DISSEMINATION}

The Human Research Ethics Committee of the University of Hong Kong reviewed and approved the study procedures and materials (reference number: EA1710040). We will give participants detailed verbal and written information on the study after their eligibility is assessed. We will provide written consent to participants before data collection. Private and confidential data will be accessed by the named researchers and will be stored with the university's secured measures for 20 years and permanently destroyed after.

We will generate academic papers and research report based on robust evidence on public transport use, built 
environment and health effects from the project. We will report anonymised and aggregated results to the scientific community through publications in academic journals. We will also present results in international conferences. The project's PI and Co-Investigators, coauthored in this paper, will coordinate all aspects of the papers, from the initial proposal to analysis plans through quality control of the manuscript. The aim is to use practice-based evidence to guide evidence-based practices in urban design and transport policy making to achieve healthy ageing. The report will provide additional design recommendations for building a more comfortable and safe walking environment to enable the elderly to use public transport. We will submit the report to government sectors for making the built environment conducive to elderly metro use. In the mean time, the report can also be a reference for future public transport system planning, in advance of the irreversible metro infrastructure investment in Hong Kong.

\section{DISCUSSION}

In this paper, we presented a study protocol for the first natural experiment study that uses a new metro line in the high-density city of Hong Kong as an intervention to investigate causal inference on elderly health. Few studies that investigated transport infrastructural modifications were concerned about the changes in public transport use brought by a new metro or have estimated the changes in physical activity and wider health outcomes of the elderly.

Although the natural experiment research design is insightful, this type of large-scale study may encounter financial and time constraints due to the high risk of postponements and other uncertainties during the infrastructure modifications. ${ }^{7}$ In our case, the schedule of opening the new metro had been postponed a year, with the new line being rearranged to two phases, and it would need another year to open the second phase. The postponed time could be a severe challenge to the follow-up survey, for example, a low retention ratio that would jeopardise the explanatory power of the study results. The treatment-control research design using longitudinal data could help rule out most confounders, but other unobservable factors could intervene during the prolonged research period. Multiple follow-ups might reduce such effects if funding allows.

Another challenge lies in analysing a cohort data set with repeated and varying measures of both exposures and outcomes. ${ }^{20}$ The new metro represents a local metrobased public transport that is continually evolving. This is especially the case in a high-density city, with a complex transport system and a 3D pedestrian network. ${ }^{31}$ We aim to use the opportunity presented by this natural experiment to investigate a more specific set of research questions linking the new metro, associated changes in the built environment and changes in bus service with public transport use, physical activity and wider health impact. Therefore, we need to adopt a flexible approach to study design due to the complex and unpredictable situations of the interventions. In addition, there are less established analytical methods for evaluation of transport infrastructural interventions. ${ }^{8}$ We need to develop our methods for understanding the effects of transport modifications and associated changes in built environment on elderly health in a high-density city.

Contributors GS, YD, MYN, JZ and CW made substantial contributions to the study conception and design. GS drafted the first manuscript. All authors revised the manuscript critically for important intellectual content. All authors have given final approval of this manuscript version, take responsibility for content and are accountable for all aspects of the work.

Funding This study is funded by the Research Grants Council (RGC) of Hong Kong (17600818). The funder had no role in study design, data collection and analysis, decision to publish, or preparation of the manuscript.

Map disclaimer The depiction of boundaries on this map does not imply the expression of any opinion whatsoever on the part of BMJ (or any member of its group) concerning the legal status of any country, territory, jurisdiction or area or of its authorities. This map is provided without any warranty of any kind, either express or implied.

\section{Competing interests None declared.}

Patient and public involvement Patients and/or the public were not involved in the design, or conduct, or reporting, or dissemination plans of this research.

Patient consent for publication Not required.

Provenance and peer review Not commissioned; externally peer reviewed.

Open access This is an open access article distributed in accordance with the Creative Commons Attribution Non Commercial (CC BY-NC 4.0) license, which permits others to distribute, remix, adapt, build upon this work non-commercially, and license their derivative works on different terms, provided the original work is properly cited, appropriate credit is given, any changes made indicated, and the use is non-commercial. See: http://creativecommons.org/licenses/by-nc/4.0/.

ORCID iD

Guibo Sun http://orcid.org/0000-0001-8493-2953

\section{REFERENCES}

1 Mackett RL, Transport TR. Social exclusion and health. J Transp Heal 2015;2:610-7.

2 Transport Department. urvey 2011 final report, 2014. Available: https://www.td.gov.hk/filemanager/en/content_4652/tcs2011_eng. pdf [Accessed 15 Mar 2021].

3 Nieuwenhuijsen MJ, Khreis $\mathrm{H}$, Verlinghieri E, et al. Transport and health: a marriage of convenience or an absolute necessity. Environ Int 2016;88:150-2.

4 Khreis H, Warsow KM, Verlinghieri E, et al. The health impacts of traffic-related exposures in urban areas: understanding real effects, underlying driving forces and co-producing future directions. $J$ Transp Health 2016;3:249-67.

5 Chatterjee K, Carey RN. Special issue on the theory, design and evaluation of behaviour change interventions in transport. Journal of Transport \& Health 2018;10:7-10.

6 Aldred R, Croft J, Goodman A. Impacts of an active travel intervention with a cycling focus in a suburban context: One-year findings from an evaluation of London's in-progress mini-Hollands programme. Transportation Research Part A: Policy and Practice 2019;123:147-69.

7 Sun G, Zhao J, Webster C, et al. New Metro system and active travel: a natural experiment. Environ Int 2020;138:105605-12.

8 Ogilvie D, Adams J, Bauman A, et al. Using natural experimental studies to guide public health action: turning the evidence-based medicine paradigm on its head. J Epidemiol Community Health 2020;74:203-8.

9 Craig P, Cooper C, Gunnell D, et al. Using natural experiments to evaluate population health interventions: new medical Research Council guidance. J Epidemiol Community Health 2012;66:1182-6.

10 Humphreys DK, Panter J, Sahlqvist S, et al. Changing the environment to improve population health: a framework for 
considering exposure in natural experimental studies. J Epidemiol Community Health 2016;70:941-6.

11 Hirsch JA, DeVries DN, Brauer M, et al. Impact of new rapid transit on physical activity: a meta-analysis. Preventive Medicine Reports 2018;10:184-90

12 Mayne SL, Auchincloss AH, Michael YL. Impact of policy and built environment changes on obesity-related outcomes: a systematic review of naturally occurring experiments. Obes Rev 2015;16:362-75.

13 Kärmeniemi M, Lankila T, Ikäheimo T, et al. The built environment as a determinant of physical activity: a systematic review of longitudinal studies and natural experiments. Ann Behav Med 2018:52:239-51.

14 Xiao C, Goryakin Y, Cecchini M. Physical activity levels and new public transit: a systematic review and meta-analysis. Am J Prev Med 2019:56:464-73.

15 Stappers NEH, Van Kann DHH, Ettema D, et al. The effect of infrastructural changes in the built environment on physical activity, active transportation and sedentary behavior - a systematic review. Health Place 2018;53:135-49.

16 Frank LD, Kuntz JL, Chapman JE, et al. The health and economic effects of light rail lines: design, methods, and protocol for a natural experiment. BMC Public Health 2019;19:1-13.

17 Heinen E, Panter J, Mackett R, et al. Changes in mode of travel to work: a natural experimental study of new transport infrastructure. Int $J$ Behav Nutr Phys Act 2015;12:81.

18 Panter J, Heinen E, Mackett R, et al. Impact of new transport infrastructure on walking, cycling, and physical activity. Am J Prev Med 2016;50:e45-53.

19 Panter J, Ogilvie D, iConnect consortium. Can environmental improvement change the population distribution of walking? $\mathrm{J}$ Epidemiol Community Health 2017;71:528-35.

20 Ogilvie D, Panter J, Guell C, et al. Health impacts of the Cambridgeshire guided Busway: a natural experimental study. Public Health Res 2016:4:1-154.

21 Heinen E, Harshfield A, Panter J, et al. Does exposure to new transport infrastructure result in modal shifts? patterns of change in commute mode choices in a four-year quasi-experimental cohort study. J Transp Health 2017;6:396-410.

22 Huang R, Moudon AV, Zhou C, et al. Light rail leads to more walking around station areas. J Transp Health 2017;6:201-8.

23 Hong A, Boarnet MG, Houston D. New light rail transit and active travel: a longitudinal study. Transportation Research Part A: Policy and Practice 2016;92:131-44.

24 Cerin E, Sit CHP, Zhang CJP, et al. Neighbourhood environment, physical activity, quality of life and depressive symptoms in Hong Kong older adults: a protocol for an observational study. BMJ Open 2016;6:e010384.

25 Cerin E, Lee K-yiu, Barnett A, et al. Walking for transportation in Hong Kong Chinese urban elders: a cross-sectional study on what destinations matter and when. Int J Behav Nutr Phys Act 2013;10:78.

26 Lu Y, Xiao Y, Ye Y. Urban density, diversity and design: is more always better for walking? A study from Hong Kong. Prev Med 2017;103S:1-5.

27 Zhang CJP, Barnett A, Sit CHP, et al. Cross-Sectional associations of objectively assessed neighbourhood attributes with depressive symptoms in older adults of an ultra-dense urban environment: the Hong Kong ALECS study. BMJ Open 2018;8:e020480-13.

28 Cerin E, Nathan A, van Cauwenberg J, et al. The neighbourhood physical environment and active travel in older adults: a systematic review and meta-analysis. Int J Behav Nutr Phys Act 2017;14:15

29 Wong K, Yeung M. Population ageing trend of Hong Kong. Econ Lett Hong Kong SAR 2019;18:1-12.

30 Wang LH. An analysis of Hong Kong transit city. Beijing: China Architecture \& Building Press, 2014.
31 Sun G, Webster C, Zhang X. Connecting the city: a three-dimensional pedestrian network of Hong Kong. Environ Plan B Urban Anal City Sci 2021;48:60-75.

32 Huang L. An analysis of Hong Kong transit city. Beijing: China Architecture \& Building Press, 2014

33 Shelton B, Karakiewicz J, Kvan T. The making of Hong Kong: from vertical to volumetric. Abingdon-on-Thames: Routledge, 2011.

34 Solomon J, Wong C, Frampton A. Cities without ground: a Hong Kong guidebook. ORO editions, 2012. Available: http:// citieswithoutground.com/

35 Zacharias J, He J. Hong Kong's urban planning experiment in enhancing pedestrian movement from underground space to the surface. Tunnelling and Underground Space Technology 2018;82:1-8.

36 Wing C, Simon K, Bello-Gomez RA. Designing difference in difference studies: best practices for public health policy research. Annu Rev Public Health 2018;39:453-69.

37 Heinen E, Wee Bvan, Panter J, et al. Residential self-selection in quasi-experimental and natural experimental studies: an extended conceptualization of the relationship between the built environment and travel behavior. J Transp Land Use 2018;11:939-59.

38 Cerin E, Sit $\mathrm{CH}$, Cheung $\mathrm{M}-\mathrm{C}$, et al. Reliable and valid news for Chinese seniors: measuring perceived neighborhood attributes related to walking. Int J Behav Nutr Phys Act 2010;7:84.

39 Steptoe A, Deaton A, Stone AA. Subjective wellbeing, health, and ageing. Lancet 2015;385:640-8.

40 Bjørnskov C. How comparable are the Gallup world Poll life satisfaction data? J Happiness Stud 2010;11:41-60.

41 Diener E, Wirtz D, Tov W, et al. New well-being measures: short scales to assess flourishing and positive and negative feelings. Soc Indic Res 2010;97:143-56.

42 Ryff CD. Happiness is everything, or is it? explorations on the meaning of psychological well-being. J Pers Soc Psychol 1989:57:1069-81.

43 Sun G, Webster C, Ni MY, et al. Measuring high-density built environment for public health research: uncertainty with respect to data, indicator design and spatial scale. Geospat Health 2018;13:35-47.

44 Dunning T. Natural experiments in the social sciences: a designbased approach. Cambridge, UK: Cambridge University Press, 2012.

45 Van Cauwenberg J, De Donder L, Clarys P, et al. Relationships between the perceived neighborhood social environment and walking for transportation among older adults. Soc Sci Med 2014;104:23-30.

46 Cummins S, Curtis S, Diez-Roux AV, et al. Understanding and representing 'place' in health research: a relational approach. Soc Sci Med 2007;65:1825-38.

47 Carpiano RM. Come take a walk with me: The "Go-Along" interview as a novel method for studying the implications of place for health and well-being. Health Place 2009;15:263-72.

48 Reichstadt J, Sengupta G, Depp CA, et al. Older adults' perspectives on successful aging: qualitative interviews. Am J Geriatr Psychiatry 2010;18:567-75.

49 Curl A, Tilley S, Van Cauwenberg J. Walking with older adults as a geographical method. In: Geographies of transport and ageing. Cham: Palgrave Macmillan, 2018: 171-95.

50 Alidoust S, Bosman C, Holden G. Talking while walking: an investigation of perceived neighbourhood walkability and its implications for the social life of older people. J Hous and the Built Environ 2018;33:133-50.

51 Van Holle V, Van Cauwenberg J, Simons D, et al. A study using walkalong interviews to explore the environmental factors related to older adults' walking for transportation. J Sci Med Sport 2012;15:S269.

52 Bengtsson M. How to plan and perform a qualitative study using content analysis. NursingPlus Open 2016;2:8-14. 